Nevşehir Bilim ve Teknoloji Dergisi Cilt 4(2) 35-43 2015

DOI: 10.17100/nevbiltek.211039

URL: http://dx.doi.org/10.17100/nevbiltek.211039

\title{
The Completeness of System of Eigenfunctions of 1D Dirac Operators
}

\author{
Hüseyin Tuna ${ }^{1 *}$, Murat Çoruh ${ }^{2}$ \\ ${ }^{1}$ Mehmet Akif Ersoy Üniversitesi, Fen Edebiyat Fakültesi, Matematik Bölümü, Burdur \\ ${ }^{2}$ Mehmet Akif Ersoy Üniversitesi, Fen Bilimleri Enstitüsü, Burdur
}

Abstract

In this paper, nonself-adjoint 1D Dirac operators in Weyl's limit-circle case are studied. Using Krein's theorems, we investigate the completeness of the system of eigenvectors and associated vectors for these operators.

Keywords: Dissipative Dirac operator, Completeness of the system of eigenvectors and associated vectors, Krein's theorem.

\section{Bir Boyutlu Dirac Operatörlerinin Özfonksiyonlar Sisteminin Tamlığı}

$\ddot{\text { Ozz }}$

Bu çalışmada Weyl limit çember durumunda kendine eş olmayan bir boyutlu Dirac operatörleri çalışılmıştır. Krein teoremleri kullanılarak, bu operatörlerin öz ve asosye vektörler sisteminin tamlığı araştırıldı.

Anahtar Kelimeler: Dissipatif Dirac Operatörleri, Öz ve asosye vektörler sisteminin tamlı̆̆ı, Krein teoremi.

*e-mail: hustuna@gmail.com 
Tuna H., Çoruh M.

\section{Introduction}

The Dirac equation is a cornerstone in the history of physics. The basic physics of relativistik quantum mechanics was formulated in the Dirac equation. It provides the origin of spin $1 / 2$ of an electron and predicts the existence of an antiparticle. The Dirac equation has been applied to realistic models like hydrogen atom [13]. We refer to the monographs [16], [25], [34] for background and further information about Dirac operators and their applications.

Dissipative operator is important part of non self adjoint operators. In the spectral analysis of a dissipative operator, we should answer the question that whether all eigenvectors and associated vectors of a dissipative operator span the whole space or not.

The first general results on completeness property of non-homogeneous string with dissipative boundary condition was obtained by Krein and Nudelman [15]. The recent publications [17]-[20] devoted to the questions of completeness and spectral synthesis for general $n \times n$ first order systems of ODE (see also references therein). In [17], [18], [20] it was shown that the completeness property for some classes of boundary conditions essentially depends on boundary values of the potential matrix and explicit conditions of the completeness were found. In particular, in [20], an example of incomplete dissipative

$2 \times 2$ Dirac operator was constructed. It was shown in [18], [19] that the resolvent of any complete dissipative Dirac type operator admits the spectral synthesis. Moreover, explicit conditions of the dissipativity and completeness of such operators were found. It is also worth to mention recent papers [5][9] devoted to the Riesz basis property for $2 \times 2$ Dirac operator (see also references therein).

In this paper we consider the one dimensional Dirac operator $L_{0}$ acting in the Hilbert space $L_{A}^{2}\left((a, b] ; \mathrm{C}^{2}\right)$ with defect index (2.2). We prove the theorems on completeness of the system of eigenvectors and associated vectors of the dissipative Dirac operator using Krein's theorems. A similar way was employed earlier in [3], [4], [11], [12], [30]-[33]..$^{\dagger}$

\section{Preliminaries}

We will consider the Dirac system

$$
l_{1}(y):=J \frac{d y(x)}{d x}+B(x) y(x)=\lambda A(x) y(x), \quad x \in I:=(a, b],-\infty \leq a<b<+\infty
$$

with singular point $a$; where $\lambda$ is a complex spectral parameter and

$$
\begin{aligned}
J & =\left(\begin{array}{cc}
0 & -1 \\
1 & 0
\end{array}\right), y(x)=\left(\begin{array}{l}
y_{1}(x) \\
y_{2}(x)
\end{array}\right), \\
A(x) & =\left(\begin{array}{ll}
a(x) & c(x) \\
c(x) & b(x)
\end{array}\right), B(x)=\left(\begin{array}{cc}
0 & q(x) \\
q(x) & 0
\end{array}\right),
\end{aligned}
$$

$A(x)>0$ ( for almost all $x \in I \quad$ ); elements of the matrices $A(x)$ and $B(x)$ are real valued,

\footnotetext{
† 2000 Mathematics Subject Classification. 34L10, 34L40.
} 
continuous functions on $I$ and $q(b) \neq 1$. Equation (2.1) is the radial wave equation for a relativistic particle. Spectral properties of (2.1) have been investigated in [1], [2], [22]-[24], [26]-[29].

To pass from the differential expression $l(y):=A^{-1}(x) l_{1}(y)(x \in I)$ to operators we introduce the Hilbert space $H:=L_{A}^{2}(I, E)\left(E:=\mathbb{C}^{2}\right)$ of vector valued functions with values in $\mathbb{C}^{2}$ and with the inner product

$$
(y, z)=\int_{a}^{b}(A(x) y(x), z(x))_{E} d x
$$

Denote by $D$ the linear set of all vectors $y \in H$ such that $y_{1}$ and $y_{2}$ are locally absolutely continuous functions on $I$ and $l(y) \in H$.

We define the operator $L$ on $D$ by the equality $L y=l y$.

For two arbitrary vectors $y, z \in D$, we have Green's formula

$$
(L y, z)-(y, L z)=[y, z]_{b}-[y, z]_{a}
$$

where $[y, z]_{x}:=W_{x}[y, \bar{z}]=y_{1}(x) \overline{z_{2}(x)}-y_{2}(x) \overline{z_{1}(x),}[y, z]_{a}=\lim _{x \rightarrow a}[y, z]_{x}$ ( see [16], [21]).

We assume that L has defect index (2.2), so that the Weyl's limit circle case holds.

Denote $u(x, \lambda)=\left(\begin{array}{l}u_{1}(x, \lambda) \\ u_{2}(x, \lambda)\end{array}\right), v(x, \lambda)=\left(\begin{array}{l}v_{1}(x, \lambda) \\ v_{2}(x, \lambda)\end{array}\right)$ the solutions of the equation

$$
l(y)=\lambda y, x \in I
$$

satisfying the initial conditions

$$
\begin{aligned}
& u_{1}(b, \lambda)=\cos \alpha, \quad u_{2}(b, \lambda)=\sin \alpha, \\
& v_{1}(b, \lambda)=-\sin \alpha \quad v_{2}(b, \lambda)=\cos \alpha .
\end{aligned}
$$

The Wronskian of the two solutions (2.3) doesn't depend on $x$, and the two solutions of this equation are linearly independent if and only if their wronskian is nonzero. It is clear that

$$
W_{x}[u, v]=W_{b}[u, v]=1, x \in I .
$$

Since $\mathrm{L}$ has defect index (2.2) $, u, v \in H$, and moreover $u, v \in D$. The solutions $u(x, \lambda)$ and $v(x, \lambda)$ form a fundamental system of (2.3) and they are entire functions of $\lambda($ see [16] ). Let $u(x)=u(x, 0)$ and $v(x)=v(x, 0)$ the solutions of the equation $l(y)=0$ satisfying the initial conditions

$$
\begin{aligned}
& u_{1}(b)=\cos \alpha, \quad u_{2}(b)=\sin \alpha, \\
& v_{1}(b)=-\sin \alpha \quad v_{2}(b)=\cos \alpha .
\end{aligned}
$$

Let us consider the functions $y \in D$ satisfying the conditions

$$
y_{1}(b) \cos \alpha+y_{2}(b) \sin \alpha=0,
$$




$$
[y, u]_{a}+h[y, v]_{a}=0,
$$

where $\operatorname{Imh}>0, \alpha \in \mathbb{R}$.

\section{Main Results}

Lemma 1. Zero is not an eigenvalue $L$.

Proof. Let $y \in D(L)$ and $L y=0$. Then

$$
J \frac{d y(x)}{d x}+B(x) y(x)=0
$$

and $y(x)=c_{1} u(x)+c_{2} v(x)$. Substituting this in the boundary conditions (2.4)-(2.5) we find that

$$
c_{1}=c_{2}=0 ; \text { i.e., } y=0 \text {. }
$$

From Lemma 1, there exist the inverse operator $L^{-1}$. In order to describe the operator $L^{-1}$ we use the Green's function method. We consider the functions $v(x)$ and $\theta(x)=u(x)+h v(x)$. These functions belong to the space $H$. Their Wronskian $W(v, \theta)=-1$.

Let

$$
G(x, t)= \begin{cases}v(x) \theta^{T}(t), & a \leq x \leq t \leq b \\ v(t) \theta^{T}(x), & a \leq t \leq x \leq b\end{cases}
$$

where ${ }^{T}$ denotes the matrix transpose. The integral operator $K$ defined by the formula

$$
K f=(G(x, t), \bar{f})_{H}(f \in H)
$$

is a compact linear operator in the space $H . \quad K$ is a Hilbert Schmidth operator. It is evident that $K=L^{-1}$. Consequently the root lineals of the operator $L$ and $K$ coincide and, therefore, the completeness in $H$ of the system of all eigenvectors and associated vectors of $L$ is equivalent to the completeness of those for $K$. Since the algebraic multiplicity of nonzero eigenvalues of a compact operator is finite, each eigenvector of $L$ may have only a finite number of linear independent associated vectors.

Definition 1 ([10]). Let $f$ be an entire function. If for each $\varepsilon>0$ there exists a finite constant $C_{\varepsilon}>0$, such that

$$
|f(\lambda)| \leq C_{\varepsilon} e^{\varepsilon|\lambda|}, \lambda \in \mathbb{C}
$$

then $f$ is called an entire function of order $\leq 1$ of growth and minimal type.

Let us define 


$$
\begin{aligned}
\tau_{1}(\lambda) & :=[\phi(x, \lambda), u(x)]_{a}, \\
\tau_{2}(\lambda) & :=[\phi(x, \lambda), v(x)]_{a}, \\
\tau(\lambda) & :=\tau_{1}(\lambda)+h \tau_{2}(\lambda) .
\end{aligned}
$$

It is clear that

$$
\sigma_{p}(L)=\{\lambda: \lambda \in \mathbb{C}, \tau(\lambda)=0\}
$$

where $\sigma_{p}(L)$ denotes the set of all eigenvalues of $L$. Since $\varphi(b, \lambda)$ is entire function of $\lambda$ of order $\leq 1$ ( see [7]), consequently, $\tau(\lambda)$ have the same property. Then $\tau(\lambda)$ is entire functions of the order $\leq 1$ of growth, and of minimal type.

Lemma 2 ([1], [2]). Let $[u, v]_{x}=1(a \leq x \leq b)$ for some real solutions $u(x)$ and $v(x)$ of equation $l(y)=0$. Then, one has the equality

$$
[y, z]_{x}=[y, u]_{x}[\bar{z}, v]_{x}-[y, v]_{x}[\bar{z}, u]_{x} .
$$

Proof. Since the functions $y_{i}(x)$ and $z_{i}(x)(i=1,2) \quad$ are real valued and $[u, v]_{x}=1(a \leq x \leq b)$, we obtain

$$
\begin{aligned}
& {[y, u]_{x}[\bar{z}, v]_{x}-[y, v]_{x}[\bar{z}, u]_{x}=\left(y_{1}(x) u_{2}(x)-y_{2}(x) u_{1}(x)\right)\left(\bar{z}_{1}(x) v_{2}(x)-\bar{z}_{2}(x) v_{1}(x)\right)} \\
& -\left(y_{1}(x) v_{2}(x)-y_{2}(x) v_{1}(x)\right)\left(\bar{z}_{1}(x) u_{2}(x)-\bar{z}_{2}(x) u_{1}(x)\right) \\
& =y_{1}(x) u_{2}(x) \bar{z}_{1}(x) v_{2}(x)-y_{1}(x) u_{2}(x) \bar{z}_{2}(x) v_{1}(x) \\
& -y_{2}(x) u_{1}(x) \bar{z}_{1}(x) v_{2}(x)+y_{2}(x) u_{1}(x) \bar{z}_{2}(x) v_{1}(x) \\
& -y_{1}(x) v_{2}(x) \bar{z}_{1}(x) u_{2}(x)+y_{1}(x) v_{2}(x) \bar{z}_{2}(x) u_{1}(x) \\
& +y_{2}(x) v_{1}(x) \bar{z}_{1}(x) u_{2}(x)-y_{2}(x) v_{1}(x) \bar{z}_{2}(x) u_{1}(x) \\
& =-y_{1}(x) u_{2}(x) \bar{z}_{2}(x) v_{1}(x)-y_{2}(x) u_{1}(x) \bar{z}_{1}(x) v_{2}(x) \\
& -y_{1}(x) v_{2}(x) \overline{z_{1}}(x) u_{2}(x)+y_{1}(x) v_{2}(x) \bar{z}_{2}(x) u_{1}(x) \\
& +y_{2}(x) v_{1}(x) \overline{z_{1}}(x) u_{2}(x) \\
& =\left(-y_{1}(x) \bar{z}_{2}(x)+y_{2}(x) \bar{z}_{1}(x)\right)\left(u_{2}(x) v_{1}(x)-u_{1}(x) v_{2}(x)\right) \\
& =[y, z]_{x} \text {. }
\end{aligned}
$$

Theorem 1. The operator $L$ is dissipative in $H$.

Proof. Let $y \in D$, then by Lagrange identity we get

$$
(L y, y)-(y, L y)=[y, y]_{b}-[y, y]_{a} .
$$

Since $y \in D$, we have

$$
[y, y]_{b}=0 .
$$


From Lemma 2 ,

$$
\begin{aligned}
{[y, y]_{a} } & =[y, u]_{a}[y, v]_{a}-[y, v]_{a}[y, u]_{a} \\
& =-2 i \operatorname{Im} h\left([y, v]_{a}\right)^{2} .
\end{aligned}
$$

From (3.5) and (3.6)

$$
\operatorname{Im}(L y, y))=\operatorname{Im} h\left([y, v]_{a}\right)^{2}
$$

and so $L$ is dissipative in $H$.

It follows from Theorem 1, all the eigenvalues of $L$ lie in the closed upper half plane $\operatorname{Im} \lambda \geq 0$.

Let us remind Krein's theorem :

Theorem 2 ([10]). The system of root vectors of a compact dissipative operator $B$ with nuclear imaginary component is complete in the Hilbert space $H$ so long as at least one of the following two conditions is fulfilled:

$$
\lim _{\rho \rightarrow \infty} \frac{n_{+}\left(\rho, B_{R}\right)}{\rho}=0, \text { or } \lim _{\rho \rightarrow \infty} \frac{n_{-}\left(\rho, B_{R}\right)}{\rho}=0,
$$

where $n_{+}\left(\rho, B_{R}\right)$ and $n_{-}\left(\rho, B_{R}\right)$ denote the numbers of the characteristic values of the real component $B_{R}$ of the operator $B$ in the intervals $[0, \rho]$ and $[-\rho, 0]$, respectively.

Theorem 3 ([14]). If the entire function $\quad f$ satisfies the condition (3.3), then

$$
\lim _{\rho \rightarrow \infty} \frac{n_{+}(\rho, f)}{\rho} \lim _{\rho \rightarrow \infty} \frac{n_{-}(\rho, f)}{\rho}=0
$$

where $n_{+}(\rho, f)$ and $n_{-}(\rho, f)$ denote the numbers of the zeros of the function $f$ in the intervals $[0, \rho]$ and $[-\rho, 0]$, respectively.

Theorem 4. The system of all root vectors of the dissipative operator $K$ is complete in $H$.

Proof. It will be sufficient to prove that the system of all root vectors of the operator $K=L^{-1}$ in (3.2) is complete in $H$. Since $\theta(x)=u(x)+h v(x)$, setting $h=h_{1}+i h_{2} \quad h_{1}, h_{2} \in \mathbb{R}$, we get from (3.2) in view of (3.1) that $K=K_{1}+i K_{2}$, where

$$
K_{1} f=\left(G_{1}(x, t), \bar{f}\right)_{H}, K_{2} f=\left(G_{2}(x, t), \bar{f}\right)_{H}
$$

and

$$
G_{1}(x, t)= \begin{cases}v(x)\left[u(t)+h_{1} v(t)\right]^{T}, & a \leq x \leq t \leq b \\ v(t)\left[u(x)+h_{1} v(x)\right]^{T}, & a \leq t \leq x \leq b\end{cases}
$$




$$
G_{2}(x, t)=h_{2} v(x) v^{T}(t), h_{2}=\operatorname{Im} h>0 .
$$

The operator $K_{1}$ is the self-adjoint Hilbert--Schmidt operator in $H$, and $K_{2}$ is the selfadjoint one dimensional operator in $H$.

Let $L_{1}$ denote the operator in $H$ generated by the differential expression $l$ and the boundary conditions

$$
\begin{aligned}
y_{1}(b) \cos \alpha+y_{2}(b) \sin \alpha & =0, \\
{[y, u]_{a}+h_{1}[y, v]_{a} } & =0, h_{1}=\operatorname{Re} h .
\end{aligned}
$$

It is easy to verify that $K_{1}$ is the inverse $L_{1}$. Further

$$
\sigma_{p}\left(L_{1}\right)=\{\lambda: \lambda \in \mathbb{C}, \Psi(\lambda)=0\}
$$

where

$$
\Psi(\lambda):=\tau_{1}(\lambda)+h_{1} \tau_{2}(\lambda)
$$

Then we find

$$
|\Psi(\lambda)| \leq C_{\varepsilon} e^{\varepsilon|\lambda|}, \forall \lambda \in \mathbb{C}
$$

Let $T=-K$ and $T=T_{1}+i T_{2}$, where $T_{1}=-K_{1}, T_{2}=-K_{2}$. The characteristic values of the operator $K_{1}$ coincide with the eigenvalues of the operator $L_{1}$. From (3.8), (3.10) and Theorem 2, we have

$$
\lim _{\rho \rightarrow \infty} \frac{m_{+}\left(\rho, T_{1}\right)}{\rho}=0, \text { or } \lim _{\rho \rightarrow \infty} \frac{m_{-}\left(\rho, T_{1}\right)}{\rho}=0,
$$

where $m_{+}\left(\rho, T_{1}\right)$ and $m_{-}\left(\rho, T_{1}\right)$ denote the numbers of the characteristic values of the real component $T_{R}=T_{1}$ in the intervals $[0, \rho]$ and $[-\rho, 0]$, respectively. Thus the dissipative operator $T$ (also of $K$ ) carries out all the conditions of Krein's theorem on completeness. The theorem is proved.

\section{References}

[1] B. P. Allahverdiev, Spectral analysis of dissipative Dirac operators with general boundary conditions, J. Math. Anal. Appl., 283, (2003), 287-303.

[2] B. P. Allahverdiev, Extensions, dilations and functional models of Dirac operators in limit-circle case. Forum Math. 17 (2005), no. 4, 591-611.

[3] E. Bairamov, E. Ugurlu, The determinants of dissipative Sturm--Liouville operators with transmission conditions, Math. Comput. Model. 53 (2011) 805-813.

[4] E. Bairamov, E. Ugurlu, Krein's theorems for a Dissipative Boundary Value Transmission Problem, Complex Anal. Oper. Theory, DOI 10.1007/s11785-011-1180-z. 
[5] A.G. Baskakov, A.V. Derbushev and A.O. Shcherbakov, The method of similar operators in the spectral analysis of non-self-adjoint Dirac operators with non-smooth potentials, Izv. Math. 75 (3) (2011), pp. 445-469.

[6] P. Djakov and B. Mityagin, Unconditional convergence of spectral decompositions of 1D Dirac operators with regular boundary conditions, Indiana Univ. Math. J., 61 (1) (2012), pp. 359-398.

[7] P. Djakov and B. Mityagin, Equiconvergence of spectral decompositions of 1D Dirac operators with regular boundary conditions, J. Approximation Theory 164 (7) (2012), pp. 879-927.

[8] P. Djakov and B. Mityagin, Criteria for existence of Riesz bases consisting of root functions of Hill and 1D Dirac operators, J. Funct. Anal. 263 (8) (2012), pp. 2300-2332.

[9] P. Djakov and B. Mityagin, Riesz bases consisting of root functions of 1D Dirac operators, Proc. Amer. Math. Soc. 141 (4) (2013), pp. 1361-1375

[10] I.C. Gohberg, M.G. Krein, Introduction to the Theory of Linear Nonselfadjoint Operators, Amer. Math. Soc., Providence, 1969

[11] G. Guseinov, Completeness theorem for the dissipative Sturm--Liouville operator, Doga-Tr. J. Math 17 (1993) 48-54.

[12] G.Sh. Guseinov, and H. Tuncay, The determinants of perturbation connected with a dissipative Sturm-Liouville operator, J. Math. Anal. Appl. 194, (1995) 39-49.

[13] H. Hasegawa, Bound states of the one dimensional Dirac equation for scalar and vector squarewell potentials, Physica E, 59 (2014), 192-201.

[14] M.G. Krein, On the indeterminate case of the Sturm-Liouville boundary problem in the interval ğ0;1Ş, Izv. Akad. Nauk SSSR Ser. Mat. 16 (4) (1952) 293-324 (in Russian).

[15] M.G. Krein and A. A Nudelman, On Some Spectral Properties of an Inhomogeneous String with Dissipative Boundary Condition, J. Operator Theory 22 (1989), 369-395

[16] B. M. Levitan and I. S. Sargsjan. Sturm-Liouville and Dirac operators. Mathematics and its Applications (Soviet Series). Kluwer Academic Publishers Group, Dordrecht, 1991 (translated from the Russian).

[17] A. A. Lunyov and M. M. Malamud, On the completeness of the root vectors for first order systems.Dokl. Math. (3) 88 (2013), 678-683.

[18] A. A. Lunyov and M. M. Malamud, On the completeness and Riesz basis property of root subspaces of boundary value problems for first order systems. Application to the Timoshenko beam model. arXiv:1401.2574 (Submitted on 11 Jan 2014).

[19] A. A. Lunyov, M. M. Malamud, On Spectral Synthesis for Dissipative Dirac Type Operators. Integr. Equ. Oper. Theory May 2014, DOI 10.1007/s00020-014-2154-9.

[20] M. M. Malamud and L. L. Oridoroga, On the completeness of root subspaces of boundary value problems for first order systems of ordinary differential equations. J. Funct. Anal. 263 (2012), 1939-1980; arXiv:0320048.

[21] M. A. Naimark, Linear Differential Operators, 2nd edn., 1968, Nauka, Moscow, English transl. of 1st. edn., 1,2 , 1969, New York. 
[22] B. W. Roos and W. C. Sangren, Spectra for a pair of singular first order differential equations, Proc. Amer. Math. Soc, 12 (1961), 468-476.

[23] B. W. Roos and W. C. Sangren, Spectra for a pair of first order differential equations, San Diego, California, General Atomic Report GA 1373, 1960.

[24] B. W. Roos and W. C. Sangren, Expansions associated with a pair of singular first-order differential equations, J. Math. Phys., 4, (1963), 999-1008.

[25] B. Thaller, The Dirac Equation (Springer, 1992).

[26] E. C. Titchmarsh, Some eigenfunction expansion formulae. Proc. London Math. Soc. 3, $11,(1961), 159-168$.

[27] E. C. Titchmarsh, A problem in relativistic quantum mechanics. Proc. London Math. Soc. 3,11,(1961), 169-192.

[28] E. C. Titchmarsh, On the nature of the spectrum in problems of relativistic quantum mechanics. Quart. J. Math. Oxford Ser. 2, 12, (1961), 227-240.

[29] E. C. Titchmarsh, On the nature of the spectrum in problems of relativistic quantum mechanics. II. Quart. J. Math. Oxford Ser. 2, 13, (1962), 181-192.2.

[30] H. Tuna, Completeness of the rootvectors of a dissipative Sturm--Liouville operators on time scales, , Applied Mathematics and Computation , 228,(2014), 108-115 .

[31] H. Tuna and A. Eryılmaz, Completeness Theorem for Discontinuous Dirac Systems, Differential Equations and Dynamical Systems , (2013), DOI: 10.1007/s12591-013-0194-2

[32] H. Tuna and A. Eryılmaz, Completeness of the system of root functions of q-Sturm-Liouville operators, Math. Commun. 19(2014), 65-73.

[33] H. Tuna and A. Eryılmaz, On the completeness of the root vectors of dissipative Dirac operators with transmission conditions, African Diaspora Journal of Mathematics ,Vol. 17,( 2), (2014), 4758.

[34] J. Weidmann, Spectral Theory of Ordinary Differential Operators, Lecture Notes in Mathematics 1258, Springer, Berlin 1987. 